\section{Shaping science education in just 100 words}

SIR - A science workshop held in Venice earlier this year under the banner of ' 100 parole per la scienza' challenged a group of one hundred 16-18-year-olds to choose 100 words that, in their collective opinion, represent crucial factors and concepts influencing trends in science today. The students were from schools all over Italy and the workshop was organized by the San Paolo Fondazione per la Scuola and Fondazione Venezia (www.100parole.it).

Their final list was assembled after an imaginative range of seminars from notable scientists and thinkers, and after extensive discussion and individual word searches of scientific works on the web and in books and journals. Here is the result, in alphabetical order: Acid/base, aggregation status, analysis, antimatter, apparatus, atmosphere, atom, bacteria, Big Bang, biodiversity, bioethics, biosphere, black hole, carbon, cell, chaos, climate, cloning, DNA, ecosystem, electricity, electron, element, energy, entropy, environment, enzyme, equilibrium, error, ethology, evolution, experiment, force, fossil, galaxy, gene, genetically modified organism, gravity, greenhouse effect, $\mathrm{H}_{2} \mathrm{O}$, heat, hydrocarbon, infinity, intelligence, Internet, life, light, link, magnetism, mass, matter, measurement, metabolism, mind, mole, molecule, motion, mutation, natural selection, nebula, neuron, organism, osmosis, particle, periodic table, $\mathrm{pH}$, photosynthesis, planet, pollution, pressure, probability, protein, pulsar, quantum, quark, radioactivity, reaction, relativity, reproduction, research, rule, science, scientific method, solution, space, species, star, stem cell, symbiosis, systems, technology, temperature, theory, time, tissue, tumour, Universe, vacuum, virus, wave.
Scientists might all learn something from this list, representing as it does how our everyday work is perceived by a small sample of bright youngsters. Alongside several words that could just as well have been listed 100 years ago (such as acid/base, magnetism, mole, scientific method), I was struck by the number of terms hinging on ethical issues in medicine and biology (6\%), the theory of evolution (5\%) and clinical terminology (5\%). The Internet too is up there among giant scientific words such as 'Universe' and 'atom'.

This thought-provoking collection of words suggests that, as working scientists, we need to care at least as much about science education as we do about publication.

\section{Marco Prunotto Nephrology Unit Laboratory, Giannina Gaslini Children's Hospital, Largo G. Gaslini 5, 16147 Genova, Italy e-mail:marco.prunotto@gmail.com}

\section{Animal research: too much faith in models clouds judgement}

SIR - Your News Feature 'Standard model' (Nature 454, 682-685; 2008) raises issues about the use of mouse models of disease that go well beyond the field of neurodegenerative disorders. As a former head of atherosclerosis research at GlaxoSmithKline laboratories, I can attest that the situation is similar for models of atherosclerosis and dyslipidaemia.

Informed users of mouse models are well aware of their limitations in relation to human pathology, so their expectations from drug studies and the relevance of these to humans are tailored accordingly. But it seems to me that, beyond this relatively small group of practitioners, wider concerns arise.

These might be viewed as a criticism of the rigour of much of

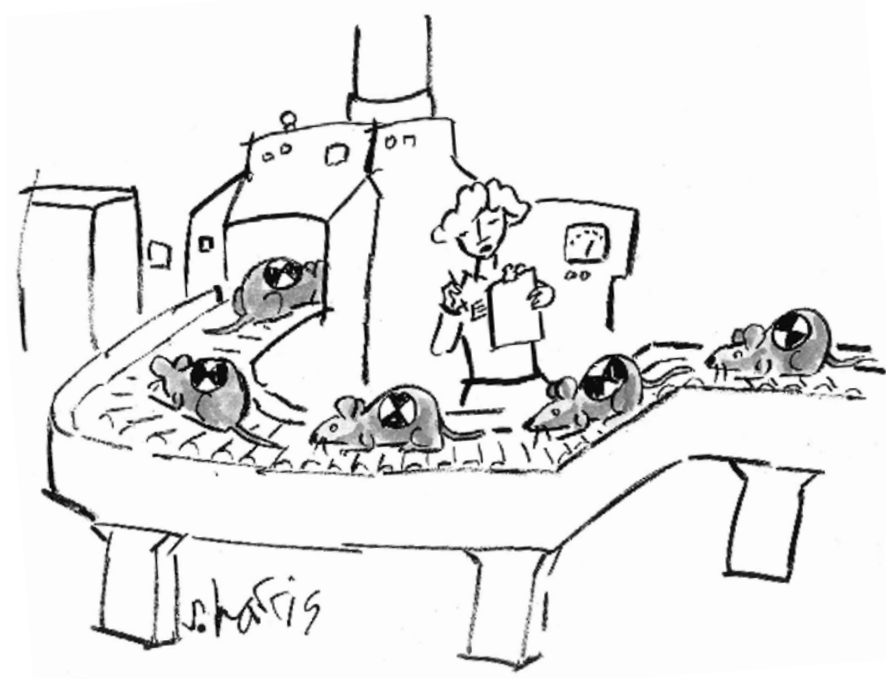

the dialogue between preclinical and clinical research. One is about overuse of the glib term "animal model of disease $X^{\prime \prime}$, which raises expectations and clouds proper interrogation of experiments. As you suggest, it is better to consider a mouse model as primarily one of mechanism and to make a reasoned extrapolation to humans from there.

This approach links in more closely with the current critical preoccupation with translational research. Another concern is one that permeates all science, namely the tendency to regard the model (in whatever form it is expressed) as being identical to its prototype, often coupled with the idea of a 'complete explanation', which is, of course, illusory.

Keith Suckling Welwyn Garden City, Herts AL8 7NH, UK e-mail: keith@suckling291.freeserve. co.uk

\section{Animal research: raise standards to protect patients}

SIR - Your News Feature 'Standard model' (Nature 454, 682-685; 2008) highlights problems in using mice as models for human neurodegenerative disease. But these pitfalls may be widespread in animal research (see, for example, P. Perel et al. Br. Med. J. 334, 197; 2007).
Animal studies are not always well designed and null results are rarely published. The standards should be raised to be comparable to those already established in clinical research. Preclinical research projects using animals should be prospectively registered and systematically reviewed. Also, they should be properly designed by using randomization, adequate sample sizes and blinding for evaluation of outcome.

Any rigorous scientific research requires investigators to set their basic hypotheses in the context of what is already published, to avoid unnecessary replication and to justify the new study. However, there is often a failure in animal research to apply these standards. This can expose research volunteers and patients to flawed and inadequate research and put biotech investors at risk of substantial long-term financial loss.

Supporting the introduction of these requirements for animal research would improve the body of evidence available to policymakers and investors with regard to human health. It would also minimize wastage of laboratory animals and improve patient safety.

Susan Green SABRE Research UK, PO Box 18653, London NW3 4UJ, UK e-mail: office@sabre.org.uk Competing interest: SABRE Research UK is a charity that raises awareness of these issues in animal research. 